J3eA, Journal sur l'enseignement des sciences et technologies de l'information et des systèmes, Volume 4, Hors-Série 1, 9 (2005)

DOI : http://dx.doi.org/10.1051/bib-j3ea:2005609

(C) EDP Sciences, 2005

Commande sans capteur mécanique des actionneurs embarqués

M. Fadel $^{1}$, R. Ruelland ${ }^{1}$, G. Gateau ${ }^{1}$, J.C. Hapiot ${ }^{1}$, P. Brodeau ${ }^{2}$ et J.P. Carayon ${ }^{2}$

${ }^{1}$ LEEI, UMR INPT-CNRS

2 rue Charles Camichel, BP 7122

F-31071 Toulouse CEDEX 7, France

${ }^{2}$ LIEBHERR AEROSPACE SA Toulouse, France 


\title{
Commande sans capteur mécanique des actionneurs embarqués
}

\author{
M. Fadel*, R. Ruelland*, G. Gateau*, JC Hapiot*, P. Brodeau**, JP Carayon** \\ *LEEI UMR INPT-CNRS 2, rue Charles Camichel 31071 Toulouse cedex 7 BP 7122 \\ ** LIEBHERR AEROSPACE SA Toulouse
}

\begin{abstract}
Résumé: Le passage à l'avion plus électrique devient une réalité et bon nombre d'actionneurs hydrauliques sont aujourd'hui remplacés par des machines tournantes électriques dimensionnées pour la réduction de volume. C'est ainsi que les vitesses nominales et les températures deviennent de plus en plus importantes alors que les capteurs mécaniques ne sont plus souhaités. Pour répondre à cela, il est nécessaire de développer des algorithmes d'observation performants et des dispositifs numériques adaptés à ces nouvelles exigences. Le travail présenté ici traite de ces différents aspects en présentant des solutions candidates.
\end{abstract}

\section{INTRODUCTION}

L'expansion du marché du transport aérien remet en cause les architectures actuelles afin de répondre aux très fortes pressions financières exercées sur le coût d'achat mais aussi le coût d'exploitation des avions.

Cette évolution se traduit par des besoins nouveaux en matière d'actionneurs, notamment pour les faibles puissances où les actionneurs électriques sont maintenant préférés aux actionneurs hydrauliques, grâce aux progrès récents accomplis dans le domaine des matériaux magnétiques et des électroniques de pilotage, en présentant des caractéristiques intéressantes, notamment sur le plan de la puissance massique. Ils constituent aujourd'hui des solutions compétitives au niveau de plusieurs équipements de type avionique. Les principaux avantages sont liés à la souplesse d'utilisation tant sur le plan de l'organisation structurelle de la fonction à réaliser (disposition des divers éléments dans l'habitacle) que par les performances intrinsèques obtenues, permettant ainsi l'introduction de fonctions de contrôle et de surveillance des systèmes.

L'ensemble se caractérise par un gain en volume, en poids et/ou en rendement qui sont autant de facteurs essentiels pour les équipements embarqués.

$\mathrm{Au}$ nombre des applications concernées, on citera les servo-vérins électriques utilisés aussi bien pour la commande des gouvernes que pour les systèmes de sensation artificielle d'effort, les électro-pompes intervenant sur le système carburant ou sur le procédé de traitement de l'air et plus simplement pour l'entraînement de divers ventilateurs.

Outre le gain en volume ces actionneurs doivent répondre aux impératifs liés à l'aéronautique (fiabilité, robustesse, rendement et performance ) et c'est ainsi que les solutions retenues conduisent à réaliser des machines à aimant fonctionnant à grande vitesse ( 30000 à 100000 tr/mn ), sans capteur mécanique. Il va sans dire que l'électronique associée devient fortement présente et doit faire l'objet d'une définition précise pour assurer la fonctionnalité satisfaisante avec une forte disponibilité tout en ne grevant pas le coût. La présente publication fait un tour d'horizon non exhaustif des solutions candidates au point de vue algorithmique et au niveau des architectures matérielles.

\section{LES ACTIONNEURS EMBARQUES.}

L'activité aéronautique est en perpétuelle recherche de réduction des coûts, qu'ils soient de développement, d'achat, d'exploitation ou de réparation.

Les actionneurs électriques ont démontré depuis plusieurs années leur totale adéquation par rapport à ces exigences lorsqu'il s'agit de remplacer des équipements hydrauliques ou pneumatiques de faible puissance. Ils auront permis de supprimer de nombreuses lignes pneumatiques, sources de problèmes d'installation et de fiabilité et à l'origine de continuelles erreurs d'identification des équipements en panne. Les performances et donc le rendement ont été améliorés à l'aide de régulations complexes et très performantes. La surveillance $\mathrm{du}$ bon fonctionnement, l'identification de pannes et le dialogue avec les calculateurs et le cockpit auront révolutionné à la fois le travail des pilotes et l'activité de maintenance. Les systèmes d'air ont ainsi bénéficié de fortes améliorations sur l'ensemble de la fonction : les vannes pneumatiques de récupération d'air sur les moteurs se sont transformées en vannes électropneumatiques contrôlées et surveillées par des calculateurs, le système de régulation de la température et de la pression s'est équipé de nombreuses vannes électriques de régulation des flux d'air, le système de pressurisation pneumatique s'est équipé d'actionneurs électriques à électronique et logiciel intégrés. Les performances ont été accrues et l'architecture du système a été fortement simplifiée ${ }^{1}$.

La nouvelle étape, initiée lors développement de l'A380, est d'introduire des actionneurs électriques sur des applications de plus forte puissance $(10 \mathrm{KW})$. Les premières applications concernées sont des fonctions secondaires ou de secours.

On peut estimer que le système d'air d'un avion de moins de cent places est équipé d'une vingtaine d'actionneurs électriques et d'autant de capteurs directement reliés aux calculateurs. 
Les recherches se concentrent à ce jour sur des fonctions plus critiques où la puissance est plus importante, toujours dans le soucis d'augmenter la performance et donc le rendement. Une des applications majeures concerne le système d'air qui pourrait tout à fait comprimer de l'air à l'aide d'un compresseur électrique et non plus en récupérant de l'air comprimé et chaud au niveau des moteurs de l'avion. Les enjeux sont multiples : suppression de vannes intégrées dans le milieu hostiles des moteurs, suppression de lignes pneumatiques et simplification de l'architecture système et un gain de rendement avion. De tels systèmes existent déjà pour des applications de «faibles puissances » $(<10 \mathrm{~kW})$ pour lesquelles seules des technologies éprouvées ont été utilisées. En particulier, la commande du moteur est effectuée à partir d'une recopie de position.

Un point fort d'amélioration a toutefois été identifié comme étant nécessaire à l'amélioration des performances techniques et industrielles. L'optimisation de la robustesse, des volumes et poids des équipements implique d'optimiser au mieux l'intégration et de concevoir des équipements capables de fonctionner à des vitesses et à des températures les plus élevées possibles. Les applications très grande vitesse sont à ce jour basées sur la commande d'un moteur à partir d'une mesure de la position angulaire du rotor. Cette recopie de position du moteur est assurée soit par des capteurs discrets de type effet hall, soit par des capteurs inductifs de type resolver. Une telle solution cause des soucis d'intégration et tend à limiter les capacités de l'actionneur au niveau vitesse et température.

Elle est aussi, par expérience, une cause de panne du fait des très fortes sollicitations induites sur l'ensemble de l'équipement. Ne plus utiliser de capteurs de position est donc une priorité pour être en mesure de satisfaire l'ensemble des exigences précédemment énoncées sans oublier les coûts qui en seront logiquement réduits.

La commande du moteur doit de ce fait s'adapter à ces contraintes et permettre de retirer tout capteur de position. L'objectif est de pouvoir piloter le moteur sans aucune recopie de position sur une très grande plage de vitesse de fonctionnement en mettant la priorité sur la robustesse de l'observateur qui devra remplacer le capteur.

\section{LA COMMANDE SANS CAPTEUR.}

Les dispositifs embarqués font apparaître un nombre important d'actionneurs nécessaires au contrôle du mouvement de divers objets ( servo-vérins, électro-pompes, dérive,...). Les cahiers des charges portent sur des régimes de vitesse à suivre et sur des trajectoires de positionnement à satisfaire, laissant place ainsi à des actionneurs divers et variés tant sur la fonction à réaliser que sur la puissance utilisée. On trouve régulièrement des machines à aimants à f.e.m. trapézoïdale pour les asservissements de vitesse et des machines à f.e.m.sinusoïdales pour le réglage de position.

$\mathrm{Du}$ fait de la montée en vitesse, la commande de ces actionneurs fait apparaître de nouveaux challenges qui impactent directement sur la nature des algorithmes utilisés et sur les organes nécessaires au calcul. La fréquence de découpage, souvent rendue fixe afin de maîtriser les pertes par commutation et les perturbations électromagnétiques, doit être suffisamment grande pour imposer des formes d'ondes correctes pour les courants, sans toutefois pénaliser les dispositifs de calcul en temps réel. A ce niveau la faible valeur de l'inductance cyclique n'est pas sans poser des problèmes aigus. La chaîne de correction fera l'objet d'attention tant sur le point $\mathrm{du}$ choix $\mathrm{du}$ modulateur (SVM, MLI régulière symétrique, ....) dans le but élargir la zone de linéarité de l'onduleur, que sur la qualité des mesures où l'accumulation de retards et/ou la présence de bruits peut s'avérer préjudiciable à la bonne maîtrise du couple. Pour les mêmes raisons il conviendra de définir des régulateurs performants et robustes (RST...), dotés de dispositifs de compensation des forces électromotrices et de procédures de limitation d'action intégrale. L'optimisation globale de cette démarche passe souvent par une réduction du nombre de capteurs électriques (courant, tension) et par l'utilisation d'une transformation se ramenant sur 2 axes. Bien entendu une évolution importante en terme de coût, de volume et de fiabilité doit pouvoir être obtenue en s'affranchissant du capteur de position, c'est-àdire en reconstruisant la position à partir des grandeurs électriques mesurées. Cette observation, associée à une procédure de démarrage, peut avantageusement remplacer le dispositif de captation. Le démarrage peut s'opérer en boucle ouverte si la caractéristique de charge le permet (couple de charge nul ou faible) ou bien être dotée d'une procédure de localisation du rotor afin d'initialiser l'algorithme de commande. Il est à noter que suivant le type de machine (pôles saillants ou pôles lisses) l'opération est plus ou moins simple à implanter.

\section{A. Reconstruction de la position du rotor.}

La connaissance de la position du rotor est indispensable à la fonction d'autopilotage c'est-à-dire à l'établissement d'un lien direct entre la fréquence de rotation et la fréquence d'alimentation. Cependant, suivant la structure de la machine, cette position doit être connue de manière continue, c'est-àdire avec une haute résolution pour une alimentation par des courants sinusoïdaux ou de manière discrète, avec une basse résolution, pour l'alimentation par des courants rectangulaires.

Pour cette dernière situation, la connaissance de la position tout les $60^{\circ}$ électriques est suffisante pour l'imposition des courants. Quelle que soit la méthode utilisée (passage par zéro des f.e.m., reconstruction des f.e.m., ...) et les mesures disponibles, la génération de signaux liés à la position rotorique espacés de $60^{\circ}$ constitue une solution allant dans le sens de la réduction du temps de calcul. Pour les machines sinusoïdales la procédure d'estimation ou d'observation doit être continue ce qui impacte directement sur les organes de calcul.

Nous allons développer ci-après différentes méthodes fournissant une évaluation de la position rotorique à partir de la mesure de 2 courants et de 2 tensions statoriques effectuée de manière synchrone. Il convient de noter que les tensions statoriques peuvent être reconstituées à partir de la tension du bus continu et de la commandes du convertisseur statique. 


\section{B. Machines à aimantation sinusoïdale.}

1) Modélisation en vue de l'observation.

\section{a) Modèle diphasé associé au stator}

Les machines employées sont essentiellement des machines à aimant permanent à pôles lisses, dont le stator est couplé en étoile. La composante homopolaire des courants statoriques ainsi que celle du flux induit par les aimants rotoriques est nulle par construction et l'utilisation de modèles triphasés n'amène pas d'informations supplémentaires pour la reconstruction de la position. Il est cependant plus courant de modéliser la machine synchrone dans un repère diphasé lié au stator communément nommé le repère $(\alpha, \beta)$. Ce modèle $(1)$ se retrouve à partir du modèle triphasé par la transformation de Concordia et représente une machine diphasée équivalente. Les grandeurs évoluent donc de manière sinusoïdale de fréquence identique à celle des grandeurs électriques au stator.

$$
\begin{gathered}
{\left[\begin{array}{c}
V_{\alpha} \\
V_{\beta}
\end{array}\right]=R s\left[\begin{array}{c}
I_{\alpha} \\
I_{\beta}
\end{array}\right]+L_{c y c} \cdot \frac{d}{d t}\left[\begin{array}{l}
I_{\alpha} \\
I_{\beta}
\end{array}\right]+\left[\begin{array}{c}
E_{\alpha}(\theta) \\
E_{\beta}(\theta)
\end{array}\right]} \\
\text { avec } \\
E_{\alpha}(\theta)=\frac{d \varphi_{\alpha r}(\theta)}{d t}, E_{\beta}(\theta)=\frac{d \varphi_{\beta r}(\theta)}{d t}
\end{gathered}
$$

Le couple électromoteur peut s'exprimer par :

$$
C_{e m}=p \cdot\left(\varphi_{\alpha r} \cdot I \beta-\varphi \beta r \cdot I \alpha\right)=p \cdot \sqrt{\frac{3}{2}} \cdot \phi_{r}(I \beta \cdot \cos (\theta)-I \alpha \cdot \sin (\theta))
$$

- $\phi$ r correspond à l' amplitude du flux rotor

- $\theta$ représente l'angle électrique

- $\quad$ représente le nombre de paires de pôles.

- $\mathrm{L}_{\mathrm{cyc}}$ représente l'inductance cyclique.

En utilisant ce type de modèle, l'information liée à la position du rotor se retrouve dans l'évolution des f.e.m. On pourra alors chercher à reconstruire ces f.e.m. ou les flux associés induits par le rotor.

Une troisième possibilité consiste à coupler les équations électriques avec le modèle mécanique afin d'introduire la position dans le vecteur d'état.

\section{b) Modèle de Park.}

L'autre modélisation souvent exploitée correspond au modèle dans le repère de Park. Ce dernier est un repère orthogonal $(d, q)$ dont l'axe $d$ est en phase avec le flux induit par le rotor. Il correspond en fait à une rotation de l'angle électrique $\theta$ du repère $(\alpha, \beta)$. La matrice de passage des grandeurs triphasées aux grandeurs dans le repère de Park est décrite ci-après (3).

$V_{d}=R I_{d}+L_{d} \frac{d I_{d}}{d t}-L_{q} \omega \cdot I_{q}$

$V_{q}=R I_{q}+L_{q} \frac{d I_{q}}{d t}+L_{d} \omega \cdot I_{d}+\sqrt{\frac{3}{2}} \phi_{r} \cdot \omega$

Le couple électromoteur s'écrit alors :

$C_{e m}=p \cdot \sqrt{\frac{3}{2}} \cdot \phi_{r} \cdot I_{q}$ si $L_{d}=L_{q}=L$

Ce modèle peut-être exploité de différentes manières, comme nous allons le voir par la suite, suivant le type d'observateur utilisé.
Le modèle décrit dans le repère $(\alpha, \beta)$ a l'avantage d'être exploitable sans tenir compte de l'équation mécanique. Cependant, ces grandeurs évoluent en fonction de la position et ont donc des dynamiques qui peuvent être relativement élevées en particulier pour les machines fonctionnant à haute vitesse. Le modèle de Park, quant à lui, a l'avantage de représenter des grandeurs ne dépendant pas de la position. Cependant, cette dernière reste nécessaire pour la transformation des mesures.

Nous proposons ci-après de développer des solutions candidates dont il faudra évaluer l'adéquation avec le cahier des charges et la qualité des mesures disponibles.

\section{2) Estimation par intégration du Flux.}

Une méthode simple d'estimation de la position, pouvant d'ailleurs être réalisée en analogique, repose sur l'intégration du flux à partir des grandeurs représentées en $\alpha$ et $\beta$ [11]. En effet, en considérant l'équation (1) nous formulons:

$\varphi_{\alpha}=L_{c y c} \cdot I_{\alpha}+\sqrt{3 / 2} \cdot \Phi_{r} \cdot \cos (\Theta)$ et $\varphi_{\beta}=L_{c y c} \cdot I_{\beta}+\sqrt{3 / 2} \cdot \Phi_{r} \cdot \sin (\Theta)(5)$

$\varphi_{\alpha}=\int\left(V_{\alpha}-R s . I_{\alpha}\right)+\varphi_{\alpha 0}$ et $\varphi_{\beta}=\int\left(V_{\beta}-R s . I_{\beta}\right)+\varphi_{\beta 0}$

Si les conditions initiales sont connues et obtenues par un précalage du rotor, une bonne estimation de la position est obtenue par:

$\hat{\theta}=\arctan \left[\frac{\varphi_{\alpha}-L_{c y c} . I_{\alpha}}{\varphi_{\beta}-L_{c y c} . I_{\beta}}\right]$

Cette méthode présente l'avantage de rester insensible aux paramètres mécaniques et de ne dépendre que de l'inductance cyclique. Pour des fonctionnements à haute vitesse c'est une caractéristique intéressante.

\section{3) Observation par le Filtre de Kalman.}

a) Principe.

Le filtre de Kalman est un reconstructeur d'état dans un environnement stochastique. Il s'agit d'un observateur d'état optimal au sens de la minimisation de la variance d'erreur entre une variable réelle et son estimée.

Le filtre de Kalman se base sur un modèle d'état linéaire stochastique (8).

$x_{k+1}=A_{k} \cdot x_{k}+B_{k} \cdot u_{k}+w_{k}$

$y_{k}=C_{k} \cdot x_{k}+v_{k}$

Dans ce modèle, $x_{k}$ représente le vecteur d'état, $u_{k}$ le vecteur d'entrée et $y_{k}$ le vecteur de mesure. Le modèle est en fait un modèle d'état déterministe auquel ont rajoute un bruit d'état $w_{k}$, et un bruit de mesure $v_{k}$. Ces bruits sont supposés être des bruits blancs gaussiens non corrélés et ayant une moyenne nulle.

Le filtre de Kalman se calcule en plusieurs étapes en utilisant la procédure suivante (Fig.1).

Le gain de Kalman $K$ est calculé pour minimiser la variance conditionnelle de l'erreur entre le vecteur d'état estimé $\hat{x}_{k}$ et le vecteur d'état réel $x_{k}$ à l'instant k, connaissant l'ensemble des mesures à l'instant $\mathrm{k}$. 


$$
V\left(\tilde{x}_{k} / y_{k}\right)=E\left(\tilde{x}_{k}^{2} / y_{k}\right)-E^{2}\left(\tilde{x}_{k} / y_{k}\right)
$$

où $\tilde{x}_{k}=x_{k}-\hat{x}_{k}$

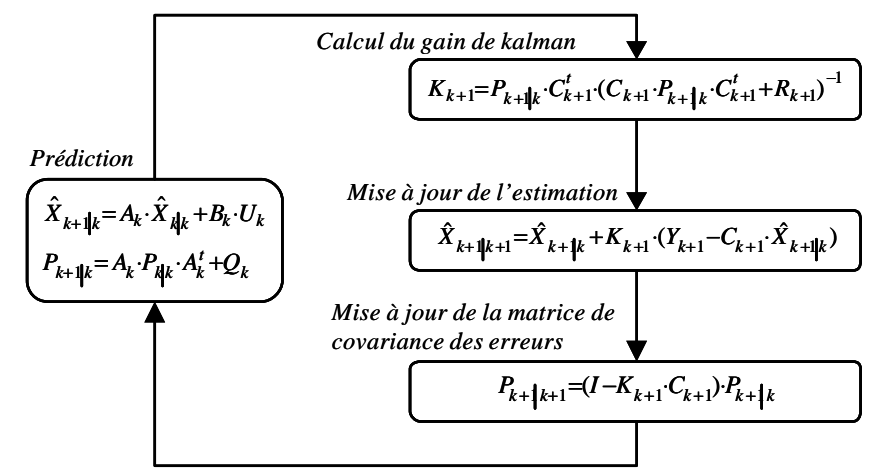

Fig. 1. Etapes de l'algorithme du filtre de Kalman récursif.

\section{b) Application.}

Que le modèle utilisé soit celui dans le repère $(\alpha, \beta)$ ou celui dans le repère de Park, il s'agit d'un modèle non-linéaire. Les principes énoncés restent les mêmes et le modèle utilisé est par contre linéarisé en utilisant un développement limité à l'ordre 1 . Le vecteur d'état choisi ici pour une représentation $(\alpha, \beta)$ est donné par $x=\left[\begin{array}{llll}I_{\alpha} & I_{\beta} & E_{\alpha} & E_{\beta}\end{array}\right]$ et $u^{t}=\left[\begin{array}{lll}V_{\alpha} & V_{\beta}\end{array}\right]$ avec les matrices suivantes :

$$
A=\left[\begin{array}{cccc}
-\frac{R}{L} & 0 & -\frac{1}{L} & 0 \\
0 & -\frac{R}{L} & 0 & -\frac{1}{L} \\
0 & 0 & \frac{\dot{\omega}}{\omega} & -\omega \\
0 & 0 & \omega & \frac{\dot{\omega}}{\omega}
\end{array}\right], B=\left[\begin{array}{cc}
\frac{1}{L} & 0 \\
0 & \frac{1}{L} \\
0 & 0 \\
0 & 0
\end{array}\right], C=\left[\begin{array}{cccc}
1 & 0 & 0 & 0 \\
0 & 1 & 0 & 0
\end{array}\right]
$$

Lors de la discrétisation, une hypothèse peut être faite sur le terme $\dot{\omega} / \omega$ de la matrice $A$ afin de simplifier la mise en œuvre.

L'utilisation du filtre de Kalman sur le modèle d'état décrit dans le repère $(\alpha, \beta)$ permet alors de reconstruire les f.e.m qui sont des grandeurs sinusoïdales. La position est déterminée en effectuant le calcul suivant :

$\theta=-\arctan \left(\frac{E_{\alpha}}{E_{\beta}}\right)(*)$

Plus classiquement le filtre de Kalman peut aussi s'employer avec le vecteur d'état $x=\left[I_{\alpha} I_{\beta} \omega \theta\right]$ en couplant les équations (1) et (5) [8].

Il convient de signaler que lorsque l'on utilise le modèle de Park il est indispensable d'utiliser l'équation mécanique car la représentation en $(d, q)$ ne véhicule pas directement l'information position.

L'utilisation du filtre de Kalman de par nature permet de prendre en compte un certain nombre d'incertitudes ce qui le rend relativement robuste. Cependant son réglage dépend de la variance du bruit d'état et du bruit de mesure, grandeurs qui sont relativement difficiles à déterminer a priori, et qui dépendent des conditions d'utilisation.

De plus, lorsque le modèle utilisé intègre l'équation mécanique, cela suppose que la charge mécanique est totalement identifiée. Si ce n'est pas le cas, le filtre de Kalman doit alors encore être étendu afin de reconstruire le couple de charge ce qui augmente d'autant la complexité du système à résoudre.

\section{4) Observation par régime glissant.}

a) Principe.

Soit le système non-linéaire d'ordre $\mathrm{n}$ suivant :

$\dot{x}=f(x, t), x \in R^{n}$ et $y=C . x, y \in R^{m}$

L'observateur peut s'écrire sous la forme :

$\hat{\dot{x}}=f(\hat{x}, t)+u_{\text {obs }}(\tilde{y}) \quad$ et $\quad \hat{y}=C . \hat{x}$

Le terme correctif en régime glissant est de la forme :

$u_{\text {obs }}=-\lambda \cdot \operatorname{sign}(\tilde{y})$ avec $(\tilde{y}=\hat{y}-y)$

où $\lambda$ est une matrice constante de dimension ( $\mathrm{n} \times \mathrm{m})$.

Le principe de l'observateur glissant utilisant $u_{\text {obs }}(\tilde{y})$ peut se diviser en deux étapes. La première étape consiste à faire converger la dynamique d'un système d'ordre $n$ en une variété d'ordre n-m, m étant le nombre de sorties mesurables. Cette variété est alors appelée surface de glissement. Plus simplement, il faut déterminer une partie de la matrice $\lambda$ afin de faire converger dans un premier temps les grandeurs estimées du modèle qui sont mesurables. On cherche donc à avoir $(\tilde{y}=\hat{y}-y) \rightarrow 0$. Cette étape correspond au régime d'atteinte, et la condition qui permet de la réaliser est dite condition de glissement. Lorsque cette étape est franchie, on obtient un système ayant une dynamique d'ordre $n-m$. La deuxième étape consiste à imposer la dynamique de ce système à l'aide des gains restant $\lambda_{i}$. Ceci peut être calculé par la méthode de Fillipov ou par une méthode similaire dite méthode de la commande équivalente. On cherche alors à annuler le reste de l'erreur d'observation qui correspond au grandeurs estimées non mesurables.

\section{b) Application.}

En théorie, ce type d'observateur peut alors être appliqué au modèle $(\alpha, \beta)$ et au modèle de Park. De plus, celui-ci fait est réputé pour sa robustesse face aux variations paramétriques. Cependant, il faut constater que ce type d'observateur à grand gain, couplé à la fonction signe qui est fortement discontinue, génère un bruit plus ou moins important sur les grandeurs observées (phénomène de chattering). Il est alors souvent nécessaire de filtrer ces grandeurs pour pouvoir reconstruire une position haute résolution exploitable dans le cas des machines à f.e.m. sinusoïdales [6], [7].

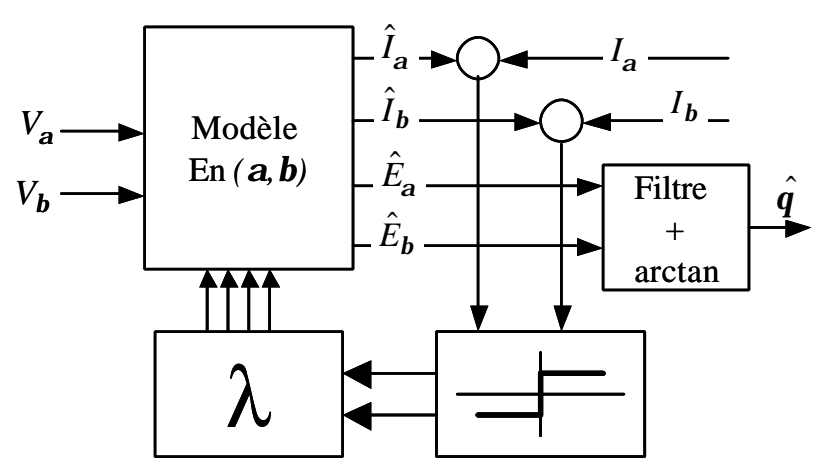

Fig. 2. Exemple d'observateur par régime glissant (modèle $(a, \beta))$. 


\section{5) Redondance analytique.}

Il s'agit ici de construire la position rotorique à partir d'une représentation sur les axes $(d, q)$ en supposant connue la position, d'où la notion de redondance. Dans le cas des machines à pôles lisses, la valeur correcte de la position se traduit par une f.e.m. nulle sur l'axe $d$ et une f.e.m. sur l'axe $q$ directement liée à la vitesse. Le bouclage de l'observateur utilise alors une action intégrale ( PI ) visant à annuler l'estimée de la f.e.m. sur l'axe $d$. Ainsi la concordance de la position estimée avec la position réelle est garantie par la cohérence du lien entre la vitesse estimée et les f.e.m. reconstruites.

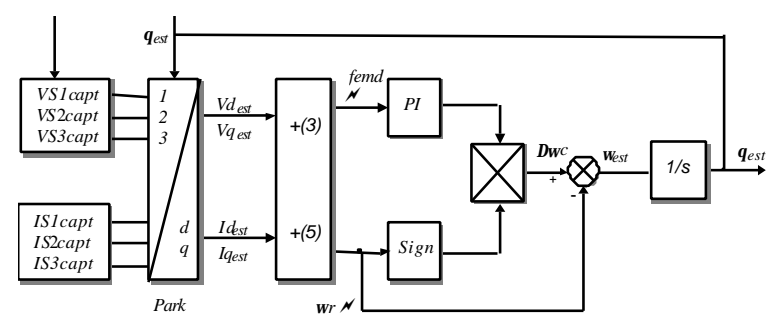

Fig. 3. Structure de l'observateur de Matsui.

Cette procédure, connue sous l'appellation d'observateur de MATSUI [2], a fait l'objet de nombreuses applications avec des modèles différents induisant des réalisations diverses plus ou moins sensibles aux bruits de mesures. Le bon fonctionnement est conditionné par un régulateur dimensionné de manière robuste c'est-à-dire faiblement sensible à la vitesse de rotation. De manière utile ce mécanisme peut être couplé à une procédure de calibrage de paramètres basée sur la valeur prise par l'action intégrale après une opération de précalage du rotor [3].

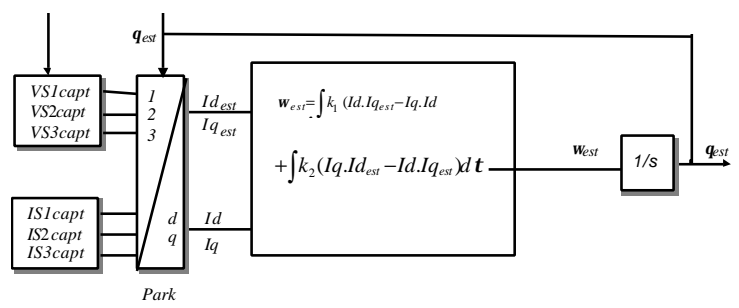

Fig. 4. Structure de l'observation par redondance analytique en intégrant l'erreur croisée sur les courants.

Selon le même principe de redondance, on peut observer la position par intégration d'une erreur croisée sur les courants mesurés et reconstruits (Fig.4). La correction robuste et stable peut reposer sur l'approche MRAS [1] synthétisée à l'aide d'hyperstabilité au sens de Popov.

\section{Machines à aimantation trapézoïdale}

\section{1) Utilisation du repère de Park étendu.}

Les machines à f.e.m. trapézoïdales font parties des machines à f.e.m. non-sinusoïdales pour lesquelles la modélisation dans le repère $(d, q)$ perd de son intérêt. Il existe cependant un repère dans lequel la modélisation garde des propriétés analogues au repère de Park [3]. Cette transformation consiste à effectuer une rotation du repère $(\alpha$, $\beta)$ non plus de l'angle électrique $\theta$ mais de l'angle $\theta+\mu(\theta)$, ou $\mu(\theta)$ permet d'aligner l'un des axes du nouveau repère avec le vecteur représentant le flux statorique induit par le rotor.

$V_{d e}=R_{s} \cdot I_{d e}+L \cdot \frac{d I_{d e}}{d t}-\omega \cdot\left(1+\frac{d \mu}{d \theta}\right) I_{q e}$

$V_{q e}=R_{s} \cdot I_{q e}+L \cdot \frac{d I_{q e}}{d t}+\omega \cdot\left(1+\frac{d \mu}{d \theta}\right) I_{d e}+\omega \cdot \phi_{r}$

Dans ce repère de Park étendu, le modèle garde un certain nombre de propriétés identiques au repère de Park classique pour les machines à f.e.m. sinusoïdales. Le couple, par exemple, ne dépend plus que d'une composante du courant statorique. Ceci peut être exploité en commande afin de minimiser les pertes par effet joules. Les propriétés de ce modèle permettent alors d'appliquer quelques méthodes d'observation vues précédemment dans le cas des machines à f.e.m. sinusoïdales, comme par exemple le principe de redondance analytique [4][5].

\section{2) Utilisation d'un repère diphasé.}

L'utilisation $\mathrm{du}$ repère $(\alpha, \beta)$ et des méthodes d'observation associées peut, quant à lui, toujours s'appliquer, mais les f.e.m. ne peuvent plus s'exprimer à l'aide de fonctions analytiques simples. Ce repère n'a donc plus grand intérêt dans le cas présent et peut être remplacé par un repère diphasé, demandant une transformation moins coûteuse en volume de calcul, comme par exemple le repère lié aux tensions composées que l'on nommera (ba-ca). Le modèle utilisé s'écrit alors sous la forme suivante :

$$
\begin{aligned}
& V_{b a}=R s \cdot\left(I_{b}-I_{a}\right)+L_{c y c} \cdot \frac{d\left(I_{b}-I_{a}\right)}{d t}+E_{b}(\theta)-E_{a}(\theta) \\
& V_{c a}=R s \cdot\left(I_{c}-I_{a}\right)+L_{c y c} \cdot \frac{d\left(I_{c}-I_{a}\right)}{d t}+E_{c}(\theta)-E_{a}(\theta)
\end{aligned}
$$

Lors de la représentation dans l'espace d'état, on supposera que la dynamique des f.e.m. est nulle. Cette hypothèse ne peut se vérifier qu'à la condition où l'échantillonnage des équations est de fréquence beaucoup plus élevée que la dynamique réelle des f.e.m. [4]. Si cette hypothèse n'est pas vérifiée, la représentation dans le repère $(b a, c a)$ ou le repère de Park étendu nécessite l'utilisation de grandeurs dépendant de la position et de la structure de la machine comme $\mu(\theta)$ ou $E_{b a}(\theta)$. Ceci nécessite la tabulation de ces grandeurs ou leur représentation par des fonctions analytiques complexes.

L'adaptation des méthodes d'observation utilisées dans le cadre des machines à f.e.m. sinusoïdales amène à reconstruire la position de manière fine. Ceci ne présente pas d'intérêt pour les machines à f.e.m. trapézoïdales qui utilisent des courants rectangulaires où il faut une information de position tous les $60^{\circ}$. Des méthodes spécifiques à ce type de machine peuvent alors être développées.

\section{3) Mesure des f.e.m. équivalentes.}

Pour les machines trapézoïdales où la connaissance périodique de la position est suffisante, on peut imaginer des dispositifs générant un signal de synchronisation permettant l'imposition cyclique de courants en adéquation avec la position rotorique. Pour chacune des phases on peut écrire : 
$V_{s 1}=R_{s 1} \cdot I_{1}+\left(L_{s}-M_{s}\right) \cdot \frac{d I_{s 1}}{d t}+E_{1}$

Ainsi la reconstruction de la f.e.m. $E_{1}$ fait intervenir la dérivée du courant statorique que l'on pourra évaluer en disposant un transformateur en série.

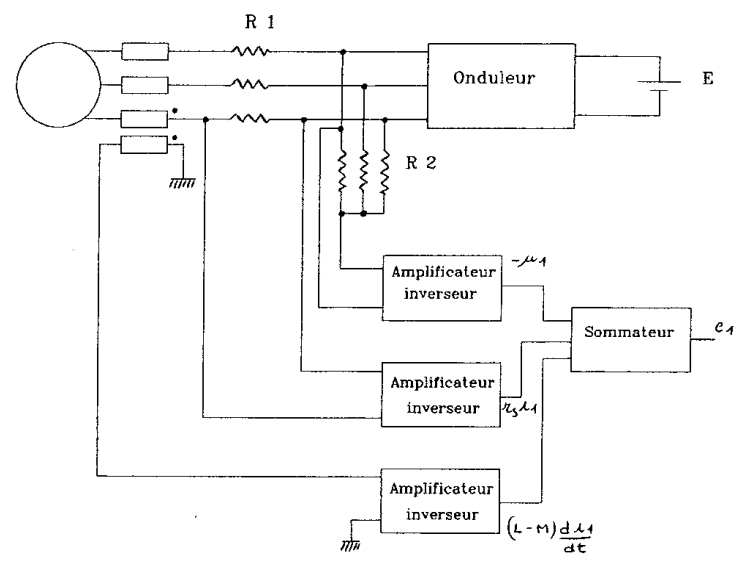

Fig. 5. Estimation des f.e.m par dérivation du courant en utilisant un transformateur.

Certes, cette solution ne va pas forcément dans le sens de la diminution de la masse, mais elle propose une solution d'intégration et de fiabilité.

Par ailleurs en considérant les 3 phases nous obtenons :

$V_{s 1}+V_{s 2}+V_{s 3}=E_{1}+E_{2}+E_{3}$.

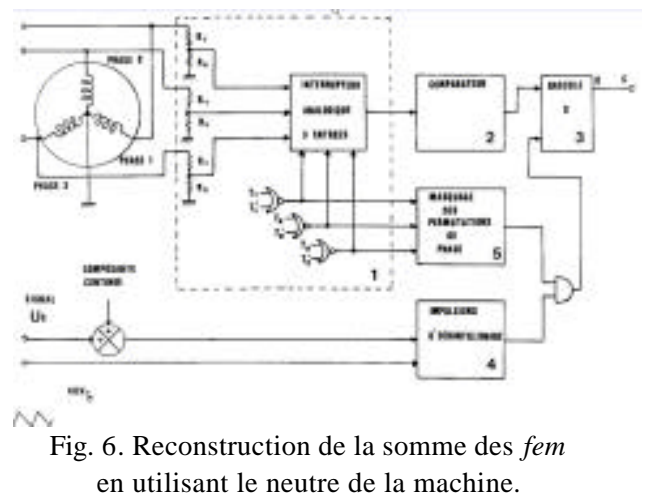

Cette somme n'est pas nulle car les f.e.m. ne sont pas sinusoïdales. S'il est possible d'accéder au point neutre de la machine nous pouvons piloter la machine avec ce signal, conformément à la figure 6 .

Nous sommes là dans le domaine des machines conçues spécifiquement induisant un surcoût éventuel. Cependant l'efficacité est au rendez-vous au même titre que la simplicité.

\section{IMPLANTATION NUMERIQUE.}

\section{A. Introduction.}

L'implantation de la commande est et restera toujours une étape importante dans le cycle de développement d'un actionneur électrique. Les choix technologiques sont toujours difficiles et doivent être guidés par la nature de l'algorithme de pilotage et d'observation. De plus, les contraintes temporelles sont beaucoup plus sévères dans le cas d'application d'actionneurs embarqués affichant des vitesses de rotation au-delà de 30000 tr/mn.
Les différents algorithmes présentés dans la partie précédente font apparaître une capacité de calculs nécessaire importante. Il est donc impératif de disposer au niveau de l'architecture de commande d'un organe performant pour effectuer des calculs lourds afin de répondre spécifiquement à ce besoin. Une solution basée sur un processeur de type DSP paraît tout à fait justifié. De plus, ce type de solution est tout à fait en accord avec les développements technologiques actuels faisant apparaître sur le marché des familles de DSP de plus en plus performantes pour des coûts de plus en plus réduits. Néanmoins, le caractère hétérogène de l'actionneur associé à son électronique de puissance (variables continues, discrètes) impose en plus, sur l'architecture de commande, la nécessité de posséder un temps de latence extrêmement court afin de pouvoir piloter les convertisseurs statiques de puissance sans problèmes. On peut par exemple parler de la génération des signaux MLI toujours délicate ou encore une commande directe des interrupteurs de puissance par une loi en modes glissants. La structure de type processeur DSP est alors prise en défaut car, comme tout système programmé, elle possède des temps de réponse pouvant devenir importants. Il est alors nécessaire de faire appel aux composants numériques programmables de type FPGA. On voit alors se dessiner une architecture mixte basée sur l'association d'un processeur de type DSP et d'un composant numérique programmable de type FPGA, chacun étant exploité suivant des critères temporels et fonctionnels qui leurs sont propres.

La répartition des différentes tâches de l'algorithme principal de régulation reste à l'entière disposition du concepteur. On effectue généralement la régulation et l'observation dans le DSP pour bénéficier de ses capacités de calculs et on laisse au FPGA la gestion des modes de marche et d'arrêt ainsi que la génération des signaux MLI. Ceci n'est pas une règle générale car il est possible de déporter l'algorithme d'observation sur la partie FPGA pour des raisons liées à la fréquence d'échantillonnage de l'observateur. En effet, pour certaines applications, celui-ci doit pouvoir être calculé à une fréquence supérieure au Méga-Hertz donc incompatible avec les capacités de calcul d'un DSP, même pour ceux de dernière génération.

\section{B. Une carte de développement versatile.}

Le dispositif de commande que nous exploitons est basé sur une carte électronique générique développée au LEEI et dénommée MPAB (Multi Purpose Application Board). Elle comprend un DSP C6711 associé au FPGA ACEX 1K100. Avec une horloge de $150 \mathrm{MHz}$, le processeur possède une capacité de calcul de 900 Mflops et 1200 Mips. Les cycles mémoire sont cadencés à $100 \mathrm{MHz}$. Le FPGA comprend l'équivalent de $10^{5}$ portes logiques, soit 4992 éléments.

Cette carte électronique (Fig. 7) a été conçue de façon à ce que le FPGA ait une place privilégiée vis à vis du DSP, dans le sens où certaines informations d'entrée/sortie passent par lui avant d'atteindre le processeur. Ainsi, 48 lignes ont été réservées aux signaux discrets permettant le pilotage de convertisseurs statiques (MLI) et/ou l'échange d'informations digitales ou numériques. Ce composant assure aussi la gestion de deux convertisseurs analogiques 
numériques «rapides» $(40 \mathrm{MHz}$ de fréquence d'échantillonnage). Les autres composants sont connectés de manière classique sur les bus du DSP. On trouve dans ce cas deux convertisseurs analogiques numériques «lents» (de 1,5 MHz à $6 \mathrm{MHz}$ de fréquence d'échantillonnage) et deux convertisseurs numériques analogiques.

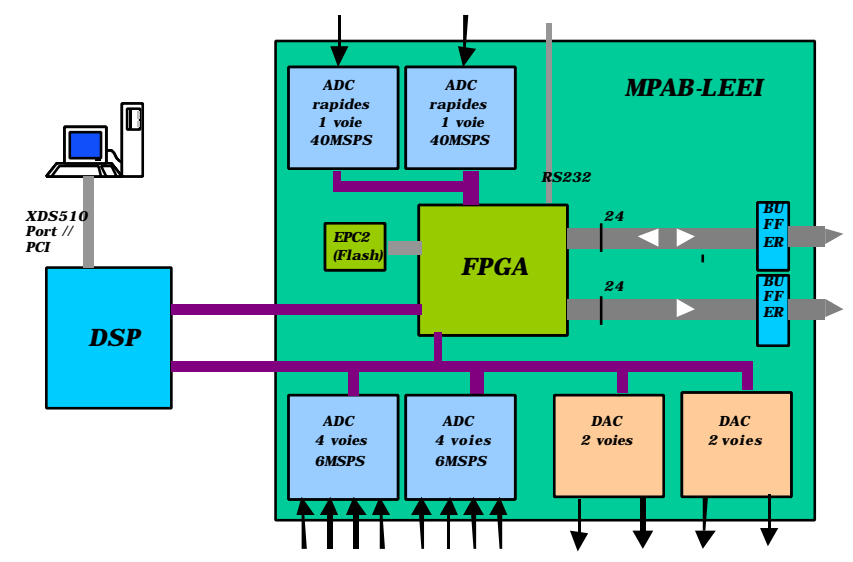

Fig. 7. Architecture du dispositif de commande basé sur la MPAB.

Cette architecture offre une très grande souplesse d'utilisation. Le FPGA étant en contact direct avec certains capteurs et actionneurs du procédé, il peut effectuer des traitements spécifiques (filtrage, détection de seuil, MLI, etc.) afin de n'échanger avec le DSP que des informations élaborées. Cette solution offre de nombreux avantages. Elle permet de décharger le processeur de certains automatismes (procédure de démarrage ou d'arrêt, MLI, etc.) pour lesquelles la logique câblée est mieux adaptée. Le FPGA peut aussi assurer des tâches fortement contraintes (gestion de défauts rapides). Enfin, le nombre de données à échanger entre ces deux composants est très limité.

Cependant, ce ne sont pas les seuls avantages. Cette architecture donne la possibilité d'utiliser le FPGA comme capteur indirect pour estimer ou observer certaines grandeurs. En effet, comme nous venons de le voir, la connaissance de la position du rotor peut nécessiter un traitement avec une haute ou une basse résolution, suivant la nature de la machine, la méthode utilisée ou encore l'amplitude de la vitesse de rotation. Tout ceci conditionne directement la fréquence à laquelle la position du rotor doit être rafraîchie, donc la période d'échantillonnage. Cependant, cette dernière est directement liée au temps de calcul donc, à processeur donné, aux équations mises en jeu par l'algorithme. Par exemple, un filtre de Kalman, relativement lourd en équations, peut se contenter d'une période d'échantillonnage égale à la période de découpage de l'onduleur. A l'inverse, la mesure desf.e.m. équivalentes, plus simple à mettre en œuvre, doit se faire de manière quasi continue. Si dans le premier cas, un traitement par le DSP donne satisfaction, il n'en est pas de même pour le deuxième.

A titre d'exemple, nous avons cherché à reconstruire la position $\mathrm{du}$ rotor à partir des forces électromotrices équivalentes. L'observateur a été implanté dans le FPGA. Ce dernier transfère la position du rotor au DSP qui, en retour, délivre les rapports cycliques nécessaires au pilotage de l'onduleur. L'architecture utilisée facilite cette opération car le FPGA dispose déjà des informations courant (CAN rapides) et tension (rapport cyclique) nécessaires aux calculs. Cette répartition a permis de satisfaire aux contraintes temporelles imposées par ce type d'observateur.

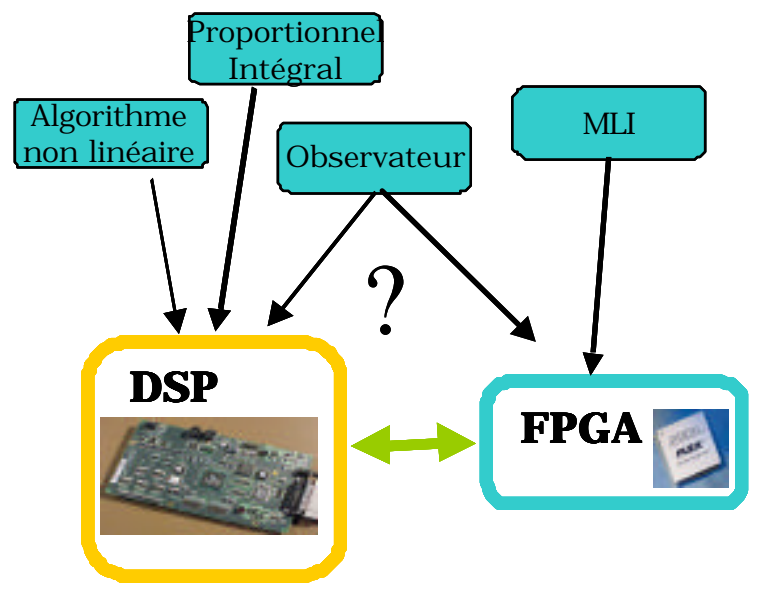

Fig. 8. Répartition possible des tâches de l'algorithme de commande.

\section{Le problème de la répartition des tâches.}

L'architecture du dispositif de commande que nous venons de présenter offre donc une grande versatilité, ce qui présente de nombreux avantages mais aussi quelques inconvénients. Le principal réside dans la répartition des tâches entre la partie câblée (FPGA) et la partie programmée (DSP). Différentes considérations liées aux contraintes fonctionnelles et temporelles sont à prendre en compte. Par exemple, le calcul de l'observateur peut s'effectuer soit au sein du DSP soit dans le FPGA. Différents essais sont souvent nécessaires avant d'aboutir à une répartition des tâches satisfaisante. Dans ce cas, pour limiter les temps de développement il peut être avantageux de faire appel à la cosimulation [10].

La démarche généralement utilisée (Fig.9) dans le développement d'un dispositif de commande consiste à définir ou choisir une architecture en se basant sur les spécifications fonctionnelles et temporelles de l'algorithme de commande. Les tâches sont ensuite réparties entre la partie logicielle (le processeur) et la partie matérielle (le FPGA). Après synthèse de ces deux parties, le seul moyen de validation dont on dispose est de procéder aux essais expérimentaux. La méthodologie que nous utilisons consiste à ajouter une étape permettant d'évaluer les performances du dispositif par simulation. C'est avec la simulation conjointe ou la co-simulation que l'on représente les fonctionnements du procédé, du processeur et du FPGA. Le procédé et son instrumentation sont simulés à l'aide d'un solveur (Saber, Matlab). Certains constructeurs fournissent le code de simulation du processeur, mais d'autres solutions peuvent être exploitées pour cela (simulation du jeu d'instructions): on utilise alors le langage C. Enfin le FPGA est simulé à l'aide du langage VHDL. 


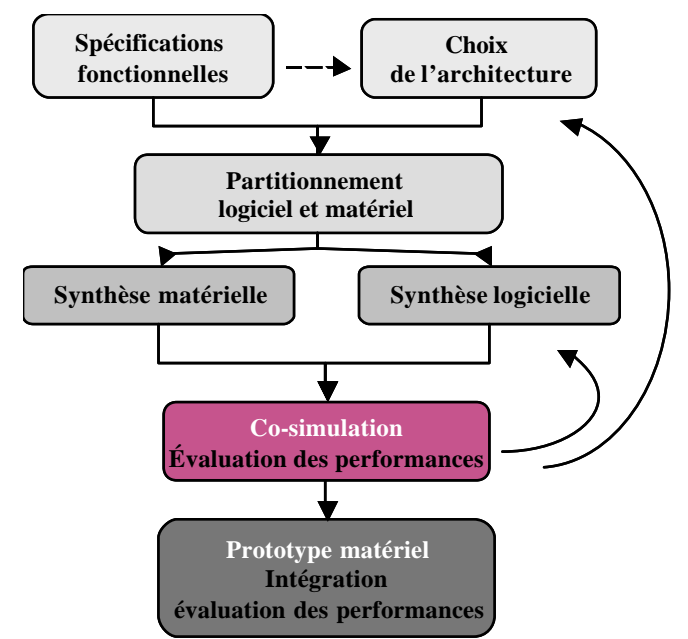

Fig. 9. Introduction de la co-simulation dans le cycle de conception d'un dispositif de commande.

Ces trois simulateurs échangent des informations par l'intermédiaire de passerelles logicielles, à l'image de ce qui se passe sur le processus réel. Cet outil permet alors d'étudier le comportement du processus avec un niveau de granularité important puisqu'il est possible de descendre au niveau d'une instruction (logiciel) ou au niveau d'une porte (matériel). Il est alors plus aisé de modifier le partitionnement matériel/logiciel ou encore, de remettre en cause l'architecture du dispositif. Différents algorithmes de commande peuvent aussi être testés. Cet outil présente encore un avantage non négligeable. Les codes de programmation du processeur et du FPGA développés pour cette co-simulation peuvent être implantés directement sur le dispositif réel. C'est le cas du FPGA où tout les constructeurs proposent un compilateur VHDL pour la programmation de leurs composants. C'est un peu moins vrai pour le processeur où quelques aménagements peuvent s'avérer nécessaires, mais l'essentiel, à savoir les calculs parfois complexes de la loi de commande, ont été validés par cette simulation. La validation expérimentale en est grandement simplifiée.

Cependant, le dispositif de commande que nous venons de présenter n'est pas toujours applicable aux actionneurs embarqués. Ce n'est pas là son objectif, mais malgré cela, il permet de valider les algorithmes de commande et le partitionnement des tâches et de définir l'architecture et les performances des composants du système qui sera embarqué. Il conviendra ensuite de prendre en compte des contraintes supplémentaires liées au domaine d'application. Nous pouvons citer par exemple la fiabilité, la CEM, la tenue aux vibrations, la tenue en température, le poids et/ou le volume, l'obsolescence des composants, le coût, etc. Il s'agit là de prendre d'intégrer des critères souvent tout aussi contraignants que ceux que l'on vient de voir, qui peuvent parfois remettre en cause l'étude aval et qu'il faudrait prendre en compte a priori dans les spécifications du système.

\section{CONCLUSION.}

L'existence d'actionneurs électromécaniques de qualité a conduit les équipementiers du domaine de l'aéronautique à utiliser de manière intensive ces solutions, tout d'abord pour des faibles puissances, puis récemment pour des applications plus importantes. Cette logique dictée par la volonté drastique de réduire les coûts de réalisation et de maintenance se poursuit de manière systématique dans le but d'améliorer encore cet état de fait. Dans ce domaine l'utilisation des machines synchrones à aimant constitue une solution qui n'est plus à justifier tant sur le plan du gain en volume et en masse que par les performances globales induites. Cependant, pour ce type de motorisation, le capteur de position nécessaire au pilotage peut constituer un point négatif sur le plan du coût ou de la fiabilité. C'est ainsi que le remplacement de cette mesure par un dispositif d'observation est maintenant envisagé et le présent travail explicite un éventail de solutions possibles à classifier suivant les applications visées. Il est clair que les exigences de réduction de volume conduisent à des actionneurs ayant des hautes vitesses de rotation contraignant fortement l'électronique de pilotage. Dans ce contexte il devient important et nécessaire d'adapter parfaitement le schéma d'observation et de contrôle, les mesures idoines en fréquence et précision avec les capacités de calcul disponibles pour réaliser un ensemble performant et robuste. In fine, cette démarche doit bien sûr être relayée par une opération d'intégration conduite en parfaite connaissance des impératifs propres à l'aéronautique.

\section{RÉFÉRENCES}

[1] Yan Liang, Yongdong Li "Speed and Position Sensorless Control of PM Synchronous" EPE'2003 Toulouse

[2] N. Matsui. "Sensorless PM Brushless DC Motor Drives". IEEE Trans. Ind. Electronics, vol. 43 n² pp.300-308, Avril 1996.

[3] D. Grenier, S. Yala, J.-P. Louis. "Définition d'extensions de la transformation de Park pour la commande de moteurs synchrones à aimants permanents montés en surface". The European Physical Journal Applied Physics, 233-246N, 1998.

[4] F. Bodin. "Commande des machines synchrones à aimants permanents et à force contre électromotrices non sinusoïdales". Thèse de Doctorat Université de Nantes, novembre 1999.

[5] L. Peyras. "Observateurs de position pour la machine synchrone avec prise en compte d'incertitudes paramétriques". Thèse de Doctorat INP de Toulouse, octobre 2003.

[6] Rédha Bensaid, "Observateurs des tensions aux bornes des capacités flottantes pour les convertisseurs multicellulaires séries". Thèse de Doctorat INP de Toulouse, avril 2001.

[7] M. Tursini, R.Petrella, F. Parasiliti. "Rotor Speed and Position Detection for PM Synchronous Motors Based on Sliding Mode Observer and Kalman Filter". Proc. of the 8th European Conference on "Power Electronics and Applications" (EPE'99), Lausanne (Svizzera) 7-9, septembre 1999.

[8] F. Colamartino. "Contribution à l'étude de la commande numérique des machines synchrones à aimants permanents". Thèse de Doctorat Université de Paris VI, décembre 1995.

[9] M. Cartignies. "Etude de l'autopilotage à partir des tensions d'une machine synchrone alimentée par onduleur de tension (contrôle en courant)". Mémoire C.N.A.M. en Electrotechnique, Toulouse, février 1990.

[10] Régis Ruelland. "Apport de la co-simulation dans la conception de l'architecture des dispositifs de commandes numérique dédiés aux systèmes électriques". Thèse de Doctorat de l'INP de Toulouse, septembre 2003.

[11] M. Boussak, R. Pilioua. "Commande Vectorielle Sans Capteur Mécanique avec Estimation de la Position Initiale des Servomoteurs Synchrones à aimants",. JTEA'96, 8-9 Novembre 1996, Hammamet, Tunisie. 